\title{
THE NEUMANN PROBLEM FOR A TYPE OF FULLY NONLINEAR COMPLEX EQUATIONS
}

\author{
WEISONG DONG AND WEI WEI
}

\begin{abstract}
In this paper we study the Neumann problem for a type of fully nonlinear second order elliptic partial differential equations on domains in $\mathbb{C}^{n}$ without any curvature assumptions on the domain.
\end{abstract}

\section{INTRODUCTION}

Let $\Omega \subset \mathbb{C}^{n}$ be a smooth domain and $\nu$ denote the unit outer normal vector on $\partial \Omega$. For a smooth function $u: \Omega \rightarrow \mathbb{R}$, we denote the complex Hessian matrix of the function $u$ by $\partial \bar{\partial} u$. Given $\psi$ a smooth nonnegative function on $\bar{\Omega} \times \mathbb{R}$ and $\varphi$ a smooth function on $\partial \Omega \times \mathbb{R}$, we study the following fully nonlinear second order partial differential equation

$$
\sigma_{k}(\Delta u \mathrm{I}-\partial \bar{\partial} u)=\psi(z, u), \text { in } \Omega,
$$

with Neumann boundary data

$$
u_{\nu}=\varphi(z, u), \text { on } \partial \Omega,
$$

where $\Delta$ is the Laplace operator, I is the $n \times n$ identity matrix and $1 \leq k \leq n-1$. For any $n \times n$ Hermitian matrix $A$, the function $\sigma_{k}$ for $1 \leq k \leq n$ is defined as $\sigma_{k}(A) \equiv \sigma_{k}(\lambda(A))=\sum \lambda_{i_{1}} \lambda_{i_{2}} \cdots \lambda_{i_{k}}$, where the sum is over $\left\{1 \leq i_{1}<\cdots<i_{k} \leq n\right\}$ and $\lambda(A)=\left(\lambda_{1}, \cdots, \lambda_{n}\right) \in \mathbb{R}^{n}$ are the eigenvalues of $A$. For convenience, we denote $\sigma_{0}(\lambda)=1$. Recall the Gårding cone $\Gamma_{k}=\left\{\lambda \in \mathbb{R}^{n}: \sigma_{i}(\lambda)>0, \forall 1 \leq i \leq k\right\}$. We say that a function $u \in C^{2}(\Omega)$ is $k$-admissible if $\lambda(\Delta u \mathrm{I}-\partial \bar{\partial} u)(z) \in \Gamma_{k}$ for every $z \in \Omega$. It is well known that at a diagonal matrix $A=\left(a_{i j}\right), \frac{\partial \sigma_{k}}{\partial a_{i j}}=\frac{\partial \sigma_{k}}{\partial \lambda_{i}} \delta_{i j}$, see Caffarelli-Nirenberg-Spruck [1. Hence, equation (1.1) is elliptic for $k$-admissible solutions, see Lemma 9 in Section 2.

Fully nonlinear equations similar to (1.1) with the particular Hermitian matrix $\Delta u \mathrm{I}-\partial \bar{\partial} u$ inside the $\sigma_{k}$ have been studied extensively. Such type of equations first comes from the study of Gauduchon conjecture. In [35] Székelyhidi-TosattiWeinkove solved a class of very general fully nonlinear equations on compact Hermitian manifolds, thereby the Gauduchon conjecture was proved as a special case of their equation. Actually, the Hermitian matrix inside the equation in [35] also involved a linear term of the gradient of $u$. Later on Tosatti-Weinkove [36] studied the Monge-Ampère type equation with a general linear gradient term inside the determinant on compact Hermitian manifolds of complex dimension $n \geq 2$. See also Yuan [39] for the case of dimension 2. The Dirichlet problem for general fully

2010 Mathematics Subject Classification. 35J60, 35B45.

Key words and phrases. Neumann problem; A priori estimate; Complex Hessian equations; Elliptic. 
nonlinear equations as in [35] has been investigated by Feng-Ge-Zheng [14 on compact Hermitian manifolds with boundaries. Chu-Jiao studied $\sigma_{k}$ type of curvature equations in [10] and Chen-Tu-Xiang studied the Hessian quotient type of curvature equations [11].

After the real Monge-Ampère equation was solved by Lions-Trudinger-Urbas in 28, the Neumann problem for complex Monge-Ampère equation on complex domains was studied by Li [26]. After that, there are few progresses on the Neumann problem for the complex fully nonlinear equations. Until recently, following the breakthrough work of Ma-Qiu [30, who solved the Neumann problem for $k$-Hessian equations on uniformly convex domains in $\mathbb{R}^{n}$, some literatures have appeared, such as Chen-Wei [6] for the complex Hessian quotient equations and Chen-Chen-MeiXiang [3] for a class of mixed complex Hessian equations. See [7] and [2] for the corresponding problems in the real situations. Comparing to the complex fully nonlinear equations, the works about the real fully nonlinear equations are more abundant. We refer to [8, 24, 23, 9, 17, 18, 32, 33, 22, 21, 4, 5, 12, 13, 16, and references therein. We remark that in the above mentioned papers, the domain is assumed to be strictly pseudoconvex, convex or mean convex. Due to the special structure of our equation, we do not need the curvature assumption on the domain. As far as the authors know, without any curvature conditions on the domain, it may be the first result about the existence of a solution to the Hessian type fully nonlinear equations. Namely, we have

Theorem 1. Suppose $\Omega \subset \mathbb{C}^{n}$ is a domain with $C^{4}$ boundary $\partial \Omega$. Let $\phi \in C^{3}(\partial \Omega)$ and $0 \leq \psi \in C^{2}(\bar{\Omega})$ be given. Then there exists a unique $k$-admissible solution $u \in C^{3, \alpha}(\bar{\Omega})$ for $0<\alpha<1$ to the Neumann problem

$$
\begin{cases}\sigma_{k}(\Delta u I-\partial \bar{\partial} u)=\psi(z), & \text { in } \Omega, \\ u_{\nu}=-\beta u+\phi(z), & \text { on } \partial \Omega,\end{cases}
$$

for any $1 \leq k \leq n-1$ and $\beta$ a positive constant, where $\nu$ is the unit outer normal vector on $\partial \Omega$.

For $k=1$ the equation is just the Poisson equation and we can drop the assumption $\psi \geq 0$. For $k \geq 2$, the equation is fully nonlinear and nonnegativity of $\psi$ is necessary for the sake of $k$-admissible solutions. The main purpose of this paper is to establish the a priori estimates for $k$-admissible solutions, and then the existence of a solution follows by the continuity method and uniqueness follows by the maximum principle. The methods here also work for real equations. Deleting the bar of index in the proof of Theorem 1 the proof is same. So the real counterpart of equation (1.4) is also solvable.

Theorem 2. Suppose $\Omega \subset \mathbb{R}^{n}$ is a domain with $C^{4}$ boundary $\partial \Omega$. Let $\phi \in C^{3}(\partial \Omega)$ and $0 \leq \psi \in C^{2}(\bar{\Omega})$ be given. Then there exists a unique $k$-admissible solution $u \in C^{3, \alpha}(\bar{\Omega})$ for $0<\alpha<1$ to the Neumann problem

$$
\begin{cases}\sigma_{k}\left(\Delta u I-D^{2} u\right)=\psi(z), & \text { in } \Omega, \\ u_{\nu}=-\beta u+\phi(z), & \text { on } \partial \Omega,\end{cases}
$$

for any $1 \leq k \leq n-1$ and $\beta$ a positive constant, where $\nu$ is the unit outer normal vector on $\partial \Omega$. 
Using Theorem 1 and the approximation argument in Lions-Trudinger-Urbas 28] (or Qiu-Xia 31 ), with the additional assumption that $\Omega$ is strictly convex, one can easily show the following theorem as Chen-Wei [6] whose proof is omitted here.

Theorem 3. Suppose $\Omega \subset \mathbb{C}^{n}$ is a $C^{4}$ strictly convex domain. Let $\phi \in C^{3}(\partial \Omega)$ and $0 \leq \psi \in C^{2}(\bar{\Omega})$ be given. Then there exist a unique constant $c$ and a unique $k$-admissible solution $u \in C^{3, \alpha}(\bar{\Omega})$ (up to a constant) for $0<\alpha<1$ to the Neumann problem

$$
\begin{cases}\sigma_{k}(\Delta u I-\partial \bar{\partial} u)=\psi(z), & \text { in } \Omega, \\ u_{\nu}=c+\phi(z), & \text { on } \partial \Omega,\end{cases}
$$

for any $1 \leq k \leq n-1$, where $\nu$ is the unit outer normal vector on $\partial \Omega$.

Remark 4. The strict convexity here is used to get the uniform gradient estimate and overcome the possible blowing up of the $C^{0}$ estimate as $\varepsilon \rightarrow 0$ for the solution $u_{\varepsilon}$ to equation (1.1) with the boundary data $u_{\nu}=-\varepsilon u+\phi(z)$ on $\partial \Omega$.

We remark here that in the above two theorems the function $\psi$ can be zero at some (or all) points in $\Omega$. This is due to our key Lemma 9 in Section 2, which is equivalent to say that the equation is strictly elliptic for $k$-admissible solutions. Hence, all the a priori estimates derived here are independent of the lower bound of $\psi$, which enable us to prove the existence result even for $\psi \geq 0$. The key lemma still holds for $k+1$-admissible solutions to the quotient type equations, i.e. $\frac{\sigma_{k}}{\sigma_{l}}$ type where $1 \leq l<k \leq n-1$. See Lemma 10. All the a priori estimates for quotient type equations can be established similarly as for the equation (1.4) using Lemma 10 instead of Lemma 9] Suppose $\Omega, \phi$ and $\psi$ are as before. Therefore, we have

Theorem 5. For any $1 \leq l<k \leq n-1$ and $\beta$ a positive constant, suppose $u \in C^{4}(\bar{\Omega})$ is a $k+1$-admissible solution to the Neumann problem

$$
\begin{cases}\frac{\sigma_{k}(\Delta u I-\partial \bar{\partial} u)}{\sigma_{l}(\Delta u I-\partial \bar{\partial} u)}=\psi(z), & \text { in } \Omega, \\ u_{\nu}=-\beta u+\phi(z), & \text { on } \partial \Omega,\end{cases}
$$

where $\nu$ is the unit outer normal vector on $\partial \Omega$. Then, we have the following a priori estimates

where $C$ depends on $\psi, \phi, \Omega, n, \beta$ and $k$.

$$
|u|_{C^{2}(\Omega)} \leq C
$$

Remark 6. The reason here we don't have existence of a solution immediately from the a priori estimate is that we don't know whether the solutions are always in $\Gamma_{k+1}$ cone when one applies the continuity method.

We give a brief outline of the proof of Theorem 1 We prove it by the standard continuity method. So we shall derive a priori estimates for $k$-admissible solutions up to second order derivatives, from which we can obtain $C^{2, \alpha}$ estimates by EvansKrylov Theorem and more higher estimates by Schauder theory. The $C^{0}$ estimate for $k$-admissible solutions can be derived easily by the maximum principle, see Lions-Trudinger-Urbas [28]. The gradient estimate is proved combining the interior estimate and near boundary estimate, i.e. estimate on $\Omega_{\mu}=\{z \in \Omega \mid d(z, \partial \Omega)<\mu\}$ for sufficiently small $\mu>0$. The second order estimate is the most difficult part. We first reduce the global second order estimate to the estimate of the double normal 
derivative on the boundary. Then, we construct barrier functions on the boundary strip $\Omega_{\mu}$ to derive upper and lower bounds for the double normal derivative on $\partial \Omega$.

The rest of this paper is organized as follows. In Section 2, we recall some properties of the elementary symmetric function $\sigma_{k}$ and prove two key lemmas, Lemma 9 and Lemma 10. In Section 3, we prove the gradient estimates, and the second order estimates are derived in Section 4. In Section 5, we prove the existence of a solution.

Acknowledgements: The first named author is supported by the National Natural Science Foundation of China, No.11801405 and No.62073236. The second named author is supported by BoXin Programme, BX20190082. Both authors would like to thank Prof. ChuanQiang Chen for careful reading and helpful suggestions.

\section{Preliminary}

We use $\sigma_{k}(\lambda \mid i)$ to denote the $k$-th elementary symmetric function with $\lambda_{i}=0$ and $\sigma_{k}(\lambda \mid i j)$ the $k$-th elementary function with $\lambda_{i}=\lambda_{j}=0$. For some useful and well known properties of the elementary symmetric function, see Li [25, LinTrudinger [29], Hou-Ma-Wu [19] and Huisken-Sinestrari [20]. Recall the following result, whose proof can be found in [29].

Lemma 7. Let $\eta=\left(\eta_{1}, \cdots, \eta_{n}\right) \in \Gamma_{k}$ and $1 \leq k \leq n$. Suppose that

$$
\eta_{1} \geq \eta_{2} \geq \cdots \geq \eta_{n}
$$

Then, we have

$$
\sigma_{k-1}(\eta \mid k) \geq c_{n, k} \sum_{i} \sigma_{k-1}(\eta \mid i)
$$

where $c_{n, k}$ is a positive constant only depending on $n$ and $k$.

The following generalized Newton-MacLaurin inequality is also used and its proof can be found in Spruck 34.

Lemma 8. For $\lambda \in \Gamma_{k}, n \geq k>l \geq 0, n \geq r>s \geq 0, k \geq r$ and $l \geq s$, we have

$$
\left(\frac{\sigma_{k}(\lambda) / C_{n}^{k}}{\sigma_{l}(\lambda) / C_{n}^{l}}\right)^{\frac{1}{k-l}} \leq\left(\frac{\sigma_{r}(\lambda) / C_{n}^{r}}{\sigma_{s}(\lambda) / C_{n}^{s}}\right)^{\frac{1}{r-s}}
$$

and the equality holds if and only if $\lambda_{1}=\cdots=\lambda_{n}$.

Let $z=\left(z_{1}, \cdots, z_{n}\right)$ be a point in $\mathbb{C}^{n}$. Sometimes we also write $z$ in real coordinates as $z=\left(t_{1}, \cdots, t_{2 n}\right)$ and $z_{i}=t_{i}+\sqrt{-1} t_{n+i}$. Given $\xi \in \mathbb{R}^{2 n}, D_{\xi} u$ denote the directional derivative of $u$ along $\xi$. For the complex variables, we use the following notations:

$$
\partial_{k} u=\frac{\partial u}{\partial z_{k}}, \partial_{\bar{k}} u=\frac{\partial u}{\partial z_{\bar{k}}}, \partial_{i \bar{j}} u=\frac{\partial^{2} u}{\partial z_{i} \partial z_{\bar{j}}}, \partial_{i \bar{j} k} u=\frac{\partial^{3} u}{\partial z_{i} \partial z_{\bar{j}} \partial z_{k}}, \cdots
$$

For simplicity, we write $u_{i}=\partial_{i} u, u_{i \bar{j}}=\partial_{i \bar{j}} u, u_{i \bar{j} k}=\partial_{i \bar{j} k} u$, and so on. It holds that

$$
|\nabla u|^{2}:=\sum_{j=1}^{n} \partial_{j} u \overline{\partial_{j} u}=\frac{1}{4}|D u|^{2} .
$$

In complex coordinates, let $\eta_{i \bar{j}} \equiv \Delta u \delta_{i j}-u_{i \bar{j}}$. With our notations, equation (1.1) can be written as

$$
F\left(u_{i \bar{j}}\right) \equiv G\left(\eta_{i \bar{j}}\right) \equiv \sigma_{k}^{\frac{1}{k}}\left(\eta_{i \bar{j}}\right)=\tilde{\psi}(z, u)
$$


where $\tilde{\psi}=\psi^{1 / k}$. Let $\lambda \equiv \lambda\left(u_{i \bar{j}}\right)=\left(\lambda_{1}, \cdots, \lambda_{n}\right)$ be the eigenvalues of $\left\{u_{i \bar{j}}\right\}$ and $\eta \equiv \lambda\left(\eta_{i \bar{j}}\right)=\left(\eta_{1}, \cdots, \eta_{n}\right)$ be the eigenvalues of $\left\{\eta_{i \bar{j}}\right\}$. Actually, $\eta_{i}=\sum_{j=1}^{n} \lambda_{j}-\lambda_{i}$. Equation (1.1) can also be written as

$$
f(\lambda) \equiv \sigma_{k}^{\frac{1}{k}}(\eta)=\tilde{\psi}(z, u) .
$$

We also introduce the following notations

$$
F^{i \bar{j}}=\frac{\partial F}{\partial u_{i \bar{j}}}, \quad F^{i \bar{j}, k \bar{l}}=\frac{\partial^{2} F}{\partial u_{i \bar{j}} \partial u_{k \bar{l}}}, \quad G^{i \bar{j}}=\frac{\partial G}{\partial \eta_{i \bar{j}}}, \quad G^{i \bar{j}, k \bar{l}}=\frac{\partial^{2} G}{\partial \eta_{i \bar{j}} \partial \eta_{k \bar{l}}} .
$$

Recall that at a point where $\left\{u_{i \bar{j}}\right\}$ is diagonal, the following identity holds

$$
F^{i \bar{j}}=f_{i} \delta_{i j}, \text { where } f_{i}=\frac{\partial f}{\partial \lambda_{i}} .
$$

This means $f_{1}, \cdots, f_{n}$ are the eigenvalues of $\left\{F^{i \bar{j}}\right\}$.

In the following, we always denote the eigenvalues of $\left\{u_{i \bar{j}}\right\}$ by $\lambda=\left(\lambda_{1}, \cdots, \lambda_{n}\right)$ with the ordering $\lambda_{1} \geq \cdots \geq \lambda_{n}$. Then, $\eta=\left(\eta_{1}, \cdots, \eta_{n}\right) \in \Gamma_{k}$ the eigenvalues of $\left\{\eta_{i \bar{j}}\right\}$ are ordered as $\eta_{1} \leq \cdots \leq \eta_{n}$, which implies $\sigma_{l}(\eta \mid 1) \geq \cdots \geq \sigma_{l}(\eta \mid n)$ for $1 \leq l \leq k-1$. Therefore, at a point $z \in \Omega$ where $\left\{u_{i \bar{j}}(z)\right\}$ is diagonal, we have

$$
F^{i \bar{i}}=\sum_{k=1}^{n} G^{k \bar{k}}-G^{i \bar{i}} \text { and } F^{1 \overline{1}} \leq \cdots \leq F^{n \bar{n}}
$$

since $G^{i \bar{i}}=\frac{1}{k} \sigma_{k}^{\frac{1}{k}-1} \sigma_{k-1}(\eta \mid i)$ and $G^{1 \overline{1}} \geq \cdots \geq G^{n \bar{n}}$. Furthermore, by the fact that $\lambda_{i}=\frac{1}{n-1} \sum_{j=1}^{n} \eta_{j}-\eta_{i}$ and $\sigma_{k}^{\frac{1}{k}}$ is homogeneous of degree one, we have

$$
\sum_{i=1}^{n} F^{i \bar{i}} u_{i \bar{i}}=\sum_{i=1}^{n} G^{i \bar{i}} \eta_{i \bar{i}}=\tilde{\psi}
$$

and

$$
\sum_{i=1}^{n} F^{i \bar{i}} u_{i \bar{i} \xi \xi}=\sum_{i=1}^{n} G^{i \bar{i}} \eta_{i \bar{i} \xi \xi} .
$$

By Lemma 7 and Lemma 8 , we can prove the following lemma which is the key to our estimates.

Lemma 9. Let $r$ be a $n \times n$ Hermitian matrix, $\lambda=\lambda(r)$ be the eigenvalues of $r$ and $\eta \in \Gamma_{k}$ where $\eta_{i}=\sum_{j} \lambda_{j}-\lambda_{i}$. Suppose $0 \leq f_{1} \leq \cdots \leq f_{n}$ are the eigenvalues of $\left\{F^{i \bar{j}}(r)\right\}$ for (2.3). If $1 \leq k \leq n-1$, we have

$$
f_{1} \geq c_{n, k} \sum_{i=1}^{n} f_{i}
$$

for some uniform positive constant $c_{n, k}$ only depending on $n$ and $k$.

Proof. We only need to prove the lemma when the matrix $r$ is diagonal. Without loss of generality, we assume $\lambda_{1}(r) \geq \cdots \geq \lambda_{n}(r)$. Hence, $\eta_{1} \leq \cdots \leq \eta_{n}$. By $f(\lambda(r))=\sigma_{k}^{\frac{1}{k}}(\eta)$, we see

$$
f_{i}=\frac{1}{k} \sigma_{k}^{\frac{1}{k}-1} \sum_{l \neq i} \sigma_{k-1}(\eta \mid l) .
$$


Since $\eta_{1} \leq \cdots \leq \eta_{n}$ and $\eta \in \Gamma_{k}$, we know

$$
\sigma_{k-1}(\eta \mid 1) \geq \sigma_{k-1}(\eta \mid 2) \geq \cdots \geq \sigma_{k-1}(\eta \mid n)
$$

By $1 \leq k \leq n-1$ and Lemma 7 we have $\sigma_{k-1}(\eta \mid 2) \geq c_{n, k} \sum_{i=1}^{n} \sigma_{k-1}(\eta \mid i)$. Hence

$$
f_{1} \geq \frac{1}{k} \sigma_{k}^{\frac{1}{k}-1} \sigma_{k-1}(\eta \mid 2) \geq \frac{c_{n, k}}{k} \sigma_{k}^{\frac{1}{k}-1} \sum_{i=1}^{n} \sigma_{k-1}(\eta \mid i)=\frac{c_{n, k}}{n-1} \sum_{i=1}^{n} f_{i}
$$

where in the last inequality we used $\sum_{i=1}^{n} f_{i}=\frac{n-1}{k} \sigma_{k}^{\frac{1}{k}-1} \sum_{i=1}^{n} \sigma_{k-1}(\eta \mid i)$.

For the quotient type equation (1.6), we can prove a similar result for $k+1$ admissible solutions. First, we can rewrite equation (1.6) as

$$
F\left(u_{i \bar{j}}\right) \equiv\left(\frac{\sigma_{k}}{\sigma_{l}}\right)^{\frac{1}{k-l}}\left(\eta_{i \bar{j}}\right)=\tilde{\psi},
$$

where $1 \leq l<k \leq n-1$ and $\tilde{\psi}=\psi^{\frac{1}{k-l}}$. Using our notations, the above equation can be written equivalently as

$$
f(\lambda) \equiv\left(\frac{\sigma_{k}}{\sigma_{l}}\right)^{\frac{1}{k-l}}(\eta)=\tilde{\psi}
$$

where $\eta \in \Gamma_{k+1}$ and $\eta_{i}=\sum \lambda_{j}-\lambda_{i}$. Similar to Lemma 9, we have

Lemma 10. Let $r$ be a $n \times n$ Hermitian matrix, $\lambda=\lambda(r)$ be the eigenvalues of $r$ and $\eta \in \Gamma_{k+1}$ where $\eta_{i}=\sum_{j} \lambda_{j}-\lambda_{i}$. Suppose $0 \leq f_{1} \leq \cdots \leq f_{n}$ are the eigenvalues of $\left\{F^{i \bar{j}}(r)\right\}$ of (2.7). If $1 \leq k \leq n-1$, we have

$$
f_{1} \geq c_{n, k, l} \sum_{i=1}^{n} f_{i}
$$

for some uniform positive constant $c_{n, k}$ only depending on $n, k$ and $l$.

Proof. We only need to prove the lemma when the matrix $r$ is diagonal. We assume $\lambda_{1}(r) \geq \cdots \geq \lambda_{n}(r)$. Hence, $\eta_{1} \leq \cdots \leq \eta_{n}$. We compute that

$$
f_{i}=\frac{1}{k-l}\left(\frac{\sigma_{k}}{\sigma_{l}}\right)^{\frac{1}{k-l}-1} \sum_{p \neq i} \frac{\sigma_{k-1}(\eta \mid p) \sigma_{l}-\sigma_{k} \sigma_{l-1}(\eta \mid p)}{\sigma_{l}^{2}} .
$$

Direct calculations show that

$$
\begin{aligned}
& \sigma_{k-1}(\eta \mid p) \sigma_{l}-\sigma_{k} \sigma_{l-1}(\eta \mid p) \\
= & \sigma_{k-1}(\eta \mid p) \sigma_{l}(\eta \mid p)-\sigma_{k}(\eta \mid p) \sigma_{l-1}(\eta \mid p) \\
= & \sigma_{k-1}(\eta \mid p) \sigma_{l}(\eta \mid p)\left(1-\alpha_{p}\right),
\end{aligned}
$$

where $\alpha_{p}$ is defined as

So we have

$$
\alpha_{p}:=\frac{\sigma_{k}(\eta \mid p)}{\sigma_{k-1}(\eta \mid p)} \frac{\sigma_{l-1}(\eta \mid p)}{\sigma_{l}(\eta \mid p)}
$$

$$
f_{i}=\frac{1}{k-l}\left(\frac{\sigma_{k}}{\sigma_{l}}\right)^{\frac{1}{k-l}-1} \sum_{p \neq i} \frac{\sigma_{k-1}(\eta \mid p) \sigma_{l}(\eta \mid p)\left(1-\alpha_{p}\right)}{\sigma_{l}^{2}} .
$$

Since $\eta \in \Gamma_{k+1}$, we see $\sigma_{k}(\eta \mid p)>0$ for every $1 \leq p \leq n$. By Newton-MacLaurin inequality (Lemma 8 with $l=s=0$ ), we have, for $1 \leq p \leq n$,

$$
0<\alpha_{p} \leq \frac{l(n-k)}{k(n-l)}<1 .
$$


Recall that $\eta_{1} \leq \cdots \leq \eta_{n}$. By Lemma 7, for any $q$ such that $1 \leq q \leq n-1$ and $\eta \in \Gamma_{q}$, we have $\sigma_{q-1}(\eta \mid 2) \geq c_{n, q} \sum_{i} \sigma_{q-1}(\eta \mid i)$. Especially, for $1 \leq l<k \leq n-1$ and $\eta \subset \Gamma_{k+1} \subset \Gamma_{k} \subset \Gamma_{l+1}$, we have

$$
\sigma_{k-1}(\eta \mid 2) \geq c_{n, k} \sum_{i=1}^{n} \sigma_{k-1}(\eta \mid i) \text { and } \sigma_{l}(\eta \mid 2) \geq c_{n, l} \sum_{i=1}^{n} \sigma_{l}(\eta \mid i) .
$$

Now we can estimate

$$
\begin{aligned}
f_{1} & \geq \frac{1}{k-l}\left(\frac{\sigma_{k}}{\sigma_{l}}\right)^{\frac{1}{k-1}-1} \frac{\sigma_{k-1}(\eta \mid 2) \sigma_{l}(\eta \mid 2)\left(1-\alpha_{2}\right)}{\sigma_{l}^{2}} \\
& \geq \frac{1}{k-l}\left(\frac{\sigma_{k}}{\sigma_{l}}\right)^{\frac{1}{k-1}-1} \frac{c_{n, k} \sum_{i=1}^{n} \sigma_{k-1}(\eta \mid i) c_{n, l} \sum \sigma_{l}(\eta \mid i)}{\sigma_{l}^{2}}\left(1-\alpha_{2}\right) \\
& \geq \frac{1}{k-l}\left(\frac{\sigma_{k}}{\sigma_{l}}\right)^{\frac{1}{k-l}-1} \frac{c_{n, k, l} \sum_{i=1}^{n} \sigma_{k-1}(\eta \mid i) \sigma_{l}(\eta \mid i)}{\sigma_{l}^{2}}\left(1-\alpha_{2}\right) \\
& \geq \frac{1}{k-l}\left(\frac{\sigma_{k}}{\sigma_{l}}\right)^{\frac{1}{k-1}-1} \frac{c_{n, k, l} \sum_{i=1}^{n} \sigma_{k-1}(\eta \mid i) \sigma_{l}(\eta \mid i)\left(1-\alpha_{i}\right)}{\sigma_{l}^{2}} \\
& =c_{n, k, l} \frac{1}{k-l}\left(\frac{\sigma_{k}}{\sigma_{l}}\right)^{\frac{1}{k-l}-1} \sum_{i=1}^{n} \frac{\sigma_{k-1}(\eta \mid i) \sigma_{l}-\sigma_{k} \sigma_{l-1}(\eta \mid i)}{\sigma_{l}^{2}}
\end{aligned}
$$

where the fourth inequality is due to (2.8) and $c_{n, k, l}$ may be different.

Note that

$$
\begin{aligned}
\sum_{i=1}^{n} f_{i} & =\frac{1}{k-l}\left(\frac{\sigma_{k}}{\sigma_{l}}\right)^{\frac{1}{k-l}-1} \sum_{i=1}^{n} \sum_{p \neq i} \frac{\sigma_{k-1}(\eta \mid p) \sigma_{l}-\sigma_{k} \sigma_{l-1}(\eta \mid p)}{\sigma_{l}^{2}} \\
& =\frac{1}{k-l}\left(\frac{\sigma_{k}}{\sigma_{l}}\right)^{\frac{1}{k-l}-1}(n-1) \sum_{i=1}^{n} \frac{\sigma_{k-1}(\eta \mid i) \sigma_{l}-\sigma_{k} \sigma_{l-1}(\eta \mid i)}{\sigma_{l}^{2}}
\end{aligned}
$$

Therefore, we obtain $f_{1} \geq \frac{c_{n, k, l}}{n-1} \sum_{i=1}^{n} f_{i}$.

Remark 11. By Newton-MacLaurin inequality (see Lemma 8 with $l=s=0$ and $r=k-1$ ), we see that

$$
\sum \sigma_{k-1}(\eta \mid i)=(n-k+1) \sigma_{k-1}(\eta) \geq(n-k+1) C_{n}^{k-1}\left(\frac{\sigma_{k}}{C_{n}^{k}}\right)^{\frac{k-1}{k}} .
$$

For equation (2.2), we obtain

$$
\sum f_{i}(\lambda) \geq \frac{n-1}{k}(n-k+1) \frac{C_{n}^{k-1}}{\left(C_{n}^{k}\right)^{\frac{k-1}{k}}}
$$

for $\lambda \in \mathbb{R}^{n}$ such that $\eta \in \Gamma_{k}$. Hence, by Lemma 9, equation (2.2) is strictly elliptic. Similar result can be shown for quotient type equation (2.7) with $\eta \in \Gamma_{k+1}$ by Lemma 8 and Lemma 10.

In the sections below, we will use $\sum_{i=1}^{n} f_{i} \geq c_{0}$ and $f_{i} \geq c_{0} \sum_{j=1}^{n} f_{j}$ for convenience, where $c_{0}$ is a positive constant depending on $n, k$ and $l$ if $l$ exists. 


\section{Gradient estimates}

In this section, we prove the $C^{1}$ estimates. We always assume that the conditions in Theorem 1 hold. We first show the following interior gradient estimate.

Theorem 12. Suppose $u \in C^{3}(\Omega)$ is a solution to (1.4) or (1.6). Assume $0 \in \Omega$ and $B_{r}(0) \subset \Omega$. Then, we have

$$
|\nabla u|(0) \leq \frac{C}{r}
$$

where $C$ depends on $|u|_{C^{0}}$ and other known data.

Proof. Consider the following auxiliary function on $B_{r}(0) \subset \Omega$,

$$
G(z)=\log |\nabla u|+h(u)+\log \zeta(z),
$$

where $\zeta(z)=r^{2}-|z|^{2}$ and $|\nabla u|^{2}=\sum_{i=1}^{n} u_{k} u_{\bar{k}}$. Assume $G$ attains its maximum at $z_{0} \in B_{r}(0)$. At $z_{0}$, we have

$$
0=G_{i}=\frac{|\nabla u|_{i}^{2}}{2|\nabla u|^{2}}+h^{\prime} u_{i}+\frac{\zeta_{i}}{\zeta}
$$

and

$$
\begin{aligned}
0 \geq & F^{i \bar{j}} G_{i \bar{j}} \\
= & F^{i \bar{j}} \frac{|\nabla u|_{i \bar{j}}^{2}}{2|\nabla u|^{2}}-F^{i \bar{j}} \frac{|\nabla u|_{i}^{2}|\nabla u|_{\bar{j}}^{2}}{2|\nabla u|^{4}} \\
& +h^{\prime} F^{i \bar{j}} u_{i \bar{j}}+h^{\prime \prime} F^{i \bar{j}} u_{i} u_{\bar{j}}+F^{i \bar{j}} \frac{\zeta_{i \bar{j}}}{\zeta}-F^{i \bar{j}} \frac{\zeta_{i} \zeta_{\bar{j}}}{\zeta^{2}} .
\end{aligned}
$$

It is immediate to see that

$$
F^{i \bar{j}} \frac{\zeta_{i \bar{j}}}{\zeta}-F^{i \bar{j}} \frac{\zeta_{i} \zeta_{\bar{j}}}{\zeta^{2}}=-\frac{\sum F^{i \bar{i}}}{\zeta}-\frac{F^{i \bar{j}} z_{\bar{i}} z_{j}}{\zeta^{2}} .
$$

By Cauchy-Schwartz inequality and (3.1), we obtain

$$
F^{i \bar{j}} \frac{|\nabla u|_{i}^{2}|\nabla u|_{\bar{j}}^{2}}{2|\nabla u|^{4}} \leq 4\left(h^{\prime}\right)^{2} F^{i \bar{j}} u_{i} u_{\bar{j}}+\frac{4}{\zeta^{2}} F^{i \bar{j}} \zeta_{i} \zeta_{\bar{j}} .
$$

Direct calculation shows

$$
F^{i \bar{j}}|\nabla u|_{i \bar{j}}^{2}=F^{i \bar{j}}\left(u_{k i} u_{\bar{k} \bar{j}}+u_{\bar{k} i} u_{k \bar{j}}\right)+u_{k} \tilde{\psi}_{\bar{k}}+u_{\bar{k}} \tilde{\psi}_{k} .
$$

We choose $h=\delta\left(u+C_{0}\right)^{2}$ where $|u|_{C^{0}} \leq C_{0}-1$ and $\delta>0$. We have

$$
h^{\prime}=2 \delta\left(u+C_{0}\right) \geq 2 \delta
$$

and

$$
h^{\prime \prime}-4\left(h^{\prime}\right)^{2}=2 \delta-16 \delta^{2}\left(u+C_{0}\right)^{2} \geq \delta
$$

for sufficiently small $\delta$. Recall that $F^{i \bar{j}} u_{i \bar{j}} \geq 0$, see (2.4). Hence, by Lemma 9, we have

$$
\begin{aligned}
0 & \geq-C+\delta F^{i \bar{j}} u_{i} u_{\bar{j}}-\frac{1}{\zeta} \sum_{i=1}^{n} F^{i \bar{i}}-\frac{5 r^{2}}{\zeta^{2}} \sum_{i=1}^{n} F^{i \bar{i}} \\
& \geq-C+\delta c_{n, k}|\nabla u|^{2} \sum_{i=1}^{n} F^{i \bar{i}}-\frac{1}{\zeta} \sum_{i=1}^{n} F^{i \bar{i}}-\frac{5 r^{2}}{\zeta^{2}} \sum_{i=1}^{n} F^{i \bar{i}} .
\end{aligned}
$$


Assume $\left|\nabla u\left(z_{0}\right)\right|^{2} \geq \frac{C}{\frac{\delta}{2} c_{n, k} c_{0}}$. We arrive at

$$
0 \geq\left(\frac{\delta c_{n, k}}{2}|\nabla u|^{2}-\frac{6 r^{2}}{\zeta^{2}}\right) \sum_{i=1}^{n} F^{i \bar{i}}
$$

from which we can derive that

$$
\zeta^{2}\left(z_{0}\right)|\nabla u|^{2}\left(z_{0}\right) \leq \frac{C r^{2}}{\delta c_{n, k}} .
$$

Therefore, by $G(0) \leq G\left(z_{0}\right)$, we obtain

$$
|\nabla u|(0) \leq \frac{C}{r}
$$

Now we prove the global gradient estimates by the following theorem.

Theorem 13. Suppose $u$ is a $C^{3} k$-admissible solution to (1.4) or (1.6). Then, we have

$$
\sup _{\Omega}|\nabla u| \leq C
$$

where $C$ depends on $|u|_{C^{0}}$ and other known data.

Proof. Denote $w=u+\varphi(z, u) d$ and $u_{\nu}=\varphi(z, u)=-\beta u+\varphi(z)$ on $\partial \Omega$. Consider the following function

$$
G(z)=\log |\nabla w|+g(d)+h(u)
$$

where $d$ is a smooth function and near boundary equals to the distance function to the boundary, $g=A d$ for a large positive constant $A$, and $h$ is a smooth function to be determined later. Suppose that $G$ attains its maximum at $z_{0} \in \bar{\Omega}$, i.e. $\max _{\bar{\Omega}} G(z)=G\left(z_{0}\right)$. We divide the proof into three cases.

Case 0: $z_{0}$ is in $\Omega_{\mu}=\{x \mid d(z, \partial \Omega) \geq \mu\}$. We can bound $|\nabla u|\left(z_{0}\right)$ by Theorem 12, i.e. we have $|\nabla u|\left(z_{0}\right) \leq \max _{\Omega_{\mu}}|\nabla u| \leq C$.

Case 1: $z_{0}$ is on the boundary $\partial \Omega$. Notice that $w_{\nu}=u_{\nu}+\varphi_{\nu} d+\varphi d_{\nu}=0$ on $\partial \Omega$, where $\nu$ is the unit outer normal vector. Hence, at $z_{0}$,

$$
\begin{aligned}
0 \leq \frac{\partial}{\partial \nu} G & =\frac{\frac{1}{4}|D w|_{\nu}^{2}}{|\nabla w|^{2}}+g^{\prime} d_{\nu}+h^{\prime} u_{\nu} \\
& =\frac{\frac{1}{2} D_{k} w D_{k \nu} w}{|\nabla w|^{2}}+g^{\prime} d_{\nu}+h^{\prime} u_{\nu} \\
& \leq 2 \sup _{\partial \Omega}\left\{\left|\Pi_{i j}\right|\right\}-A+h^{\prime} \varphi\left(z_{0}, u\right),
\end{aligned}
$$

which yields a contradiction to the larger choice of

$$
A=2 \sup _{\partial \Omega}\left\{\left|\Pi_{i j}\right|\right\}+\sup _{\Omega}\left|h^{\prime}\right||\varphi|+1 .
$$

Case 2: $z_{0}$ is in $\Omega \backslash \Omega_{\mu}$. Differentiate $G$ at $z_{0}$ once to obtain that

$$
0=G_{i}\left(z_{0}\right)=\frac{|\nabla w|_{i}^{2}}{|\nabla w|^{2}}+g^{\prime} d_{i}+h^{\prime} u_{i}
$$


and a second time to get that

$$
\begin{aligned}
0 & \geq F^{i \bar{j}} G_{i \bar{j}} \\
& =F^{i \bar{j}} \frac{|\nabla w|_{i \bar{j}}^{2}}{|\nabla w|^{2}}-F^{i \bar{j}} \frac{|\nabla w|_{i}^{2}|\nabla w|_{\bar{j}}^{2}}{|\nabla w|^{4}}+g^{\prime} F^{i \bar{j}} d_{i \bar{j}}+h^{\prime} F^{i \bar{j}} u_{i \bar{j}}+h^{\prime \prime} F^{i \bar{j}} u_{i} u_{\bar{j}} \\
& =F^{i \bar{j}} \frac{|\nabla w|_{i \bar{j}}^{2}}{|\nabla w|^{2}}-F^{i \bar{j}}\left(g^{\prime} d_{i}+h^{\prime} u_{i}\right)\left(g^{\prime} d_{\bar{j}}+h^{\prime} u_{\bar{j}}\right)+g^{\prime} F^{i \bar{j}} d_{i \bar{j}}+h^{\prime} F^{i \bar{j}} u_{i \bar{j}}+h^{\prime \prime} F^{i \bar{j}} u_{i} u_{\bar{j}} \\
& \geq F^{i \bar{j}} \frac{|\nabla w|_{i \bar{j}}^{2}}{|\nabla w|^{2}}-2\left(g^{\prime}\right)^{2} F^{i \bar{j}} d_{i} d_{\bar{j}}+\left(h^{\prime \prime}-2\left(h^{\prime}\right)^{2}\right) F^{i \bar{j}} u_{i} u_{\bar{j}}+g^{\prime} F^{i \bar{j}} d_{i \bar{j}}+h^{\prime} F^{i \bar{j}} u_{i \bar{j}},
\end{aligned}
$$

where in the last inequality we used Cauchy-Schwarz inequality.

Now let us deal with $F^{i \bar{j}} \frac{|\nabla w|_{i \bar{j}}^{2}}{|\nabla w|^{2}}$. First, we compute that

$$
\begin{aligned}
F^{i \bar{j}}|\nabla w|_{i \bar{j}}^{2} & =F^{i \bar{j}}\left(w_{k} w_{\bar{k} i \bar{j}}+w_{k i} w_{\bar{k} \bar{j}}+w_{k i \bar{j}} w_{\bar{k}}+w_{k \bar{j}} w_{\bar{k} i}\right) \\
& =w_{k} F^{i \bar{j}} w_{\bar{k} i \bar{j}}+F^{i \bar{j}} w_{k i \bar{j}} w_{\bar{k}}+F^{i \bar{j}}\left(w_{k i} w_{\bar{k} \bar{j}}+w_{k \bar{j}} w_{\bar{k} i}\right) .
\end{aligned}
$$

Recall that $\varphi(z, u)=-\beta u+\phi(z)$. We have

$$
\begin{aligned}
F^{i \bar{j}} w_{\bar{k} i \bar{j}}= & F^{i \bar{j}} u_{\bar{k} i \bar{j}}+F^{i \bar{j}}(\varphi d)_{\bar{k} i \bar{j}} \\
= & \tilde{\psi}_{\bar{k}}+F^{i \bar{j}}[(-\beta u+\phi(z)) d]_{\bar{k} i \bar{j}} \\
= & F^{i \bar{j}}\left(-\beta u_{\bar{k} i} d_{\bar{j}}-\beta u_{\bar{k} \bar{j}} d_{i}-\beta u_{i \bar{j}} d_{\bar{k}}-\beta u_{\bar{k}} d_{i \bar{j}}-\beta u_{i} d_{\bar{k} \bar{j}}\right. \\
& \left.-\beta u_{\bar{j}} d_{\bar{k} i}-\beta u d_{\bar{k} i \bar{j}}\right)+F^{i \bar{j}}(\phi(x) d)_{\bar{k} i \bar{j}}+\tilde{\psi}_{\bar{k}}(1-\beta d),
\end{aligned}
$$

and then by Cauchy-Schwarz inequality we get

$$
\begin{aligned}
& F^{i \bar{j}} w_{k i \bar{j}} w_{\bar{k}}+w_{k} F^{i \bar{j}} w_{\bar{k} i \bar{j}} \\
\geq & \left(\tilde{\psi}_{\bar{k}} w_{k}+\tilde{\psi}_{k} w_{\bar{k}}\right)(1-\beta d)-\varepsilon F^{i \bar{j}}\left(u_{k i} u_{\bar{k} \bar{j}}+u_{k \bar{j}} u_{\bar{k} i}\right) \\
& -C_{\varepsilon} \sum_{i=1}^{n} F^{i \bar{i}}\left(|\nabla u|^{2}+|\nabla u|\right) .
\end{aligned}
$$

Direct calculation shows that

$$
\begin{aligned}
& F^{i \bar{j}}\left(w_{k i} w_{\bar{k} \bar{j}}+w_{k \bar{j}} w_{\bar{k} i}\right) \\
= & F^{i \bar{j}}(u(1-\beta d)+\phi(x) d)_{k i}(u(1-\beta d)+\phi(x) d)_{\bar{k} \bar{j}} \\
& +F^{i \bar{j}}(u(1-\beta d)+\phi(x) d)_{\bar{k} i}(u(1-\beta d)+\phi(x) d)_{k \bar{j}} \\
\geq & (1-\beta d)^{2} F^{i \bar{j}}\left(u_{k i} u_{\bar{k} \bar{j}}+u_{k \bar{j}} u_{\bar{k} i}\right)(1-\varepsilon)-C_{\varepsilon} \sum_{i=1}^{n} F^{i \bar{i}}\left(|\nabla u|^{2}+|\nabla u|+1\right),
\end{aligned}
$$

where in the last inequality we used Cauchy-Schwarz inequality. By (3.3), (3.4) and (3.5), for $\mu$ chosen sufficiently small, we obtain

$$
F^{i \bar{j}}|\nabla w|_{i \bar{j}}^{2} \geq-C|\nabla u|^{2}-C \sum_{i=1}^{n} F^{i \bar{i}}\left(|\nabla u|^{2}+|\nabla u|+1\right) .
$$


From (3.2), we have

$$
\begin{aligned}
0 \geq & -C-C \sum_{i=1}^{n} F^{i \bar{i}}-2\left(g^{\prime}\right)^{2} F^{i \bar{j}} d_{i} d_{\bar{j}} \\
& +\left(h^{\prime \prime}-2\left(h^{\prime}\right)^{2}\right) F^{i \bar{j}} u_{i} u_{\bar{j}}+g^{\prime} F^{i \bar{j}} d_{i \bar{j}}+h^{\prime} F^{i \bar{j}} u_{i \bar{j}} .
\end{aligned}
$$

We choose $h=\delta\left(u+C_{0}\right)^{2}$ where $|u|_{C^{0}} \leq C_{0}-1$ and $\delta>0$. We have

$$
h^{\prime}>2 \delta \text { and } h^{\prime \prime}-2\left(h^{\prime}\right)^{2}>\delta
$$

for $\delta>0$ sufficiently small. By the fact that $F^{i \bar{j}} u_{i} u_{\bar{j}} \geq c_{0} \sum_{i=1}^{n} F^{i \bar{i}}|\nabla u|^{2}, \sum_{i=1}^{n} F^{i \bar{i}} \geq$ $c_{0}$, and $F^{i \bar{j}} u_{i \bar{j}} \geq 0$, (3.6) yields that

$$
|\nabla u|^{2} \leq C
$$

Remark 14. One can show that the global $C^{1}$ estimate still holds for $k$-admissible solutions to (1.4) with the right hand term $\psi(z, u, D u)$ by the proof with minor changes.

\section{SECOND ORDER ESTimates}

In this section, we prove the second order estimates. We first reduce the second order estimates to the double normal derivative on the boundary by the following theorem.

Theorem 15. Suppose $u$ is a $C^{4} k$-admissible solution to (1.4) or (1.6). Then, we have

$$
\sup _{(z, \xi) \in \Omega \times S^{2 n-1}} D_{\xi \xi} u(z) \leq C\left(1+\sup _{\partial \Omega}\left|D_{\nu \nu} u\right|\right),
$$

where $C$ depends on $|u|_{C^{1}}$ and other known data.

Proof. Define $h=e^{-A r}$, where $r$ is a function in $C^{2}(\bar{\Omega})$ with $\left.r\right|_{\partial \Omega}=0$ and $D_{\nu} r=1$ on $\partial \Omega, A=1+2 \max _{\partial \Omega}\left\{\left|\Pi_{i j}\right|\right\}+|\beta|$ is a constant, and $\Pi_{i j}$ is the second fundamental form of the boundary. We adopt the following auxiliary function

$$
\Phi(z, \zeta)=h(r)\left(D_{\zeta \zeta} u-v(z, \zeta)\right)+|\nabla u|^{2}
$$

where $v(x, \zeta)=2\langle\zeta, \nu\rangle\left\langle\zeta^{\prime}, D \phi-\beta D u-D_{l} u D \nu^{l}\right\rangle \equiv a^{l} D_{l} u+b, \zeta^{\prime}=\zeta-\langle\zeta, \nu\rangle \nu$, $a^{l}=-2\langle\zeta, \nu\rangle\left\langle\zeta^{\prime}, D \nu^{l}\right\rangle-2 \beta\langle\zeta, \nu\rangle\left(\zeta^{\prime}\right)^{l}, b=2\langle\zeta, \nu\rangle\left\langle\zeta^{\prime}, D \phi\right\rangle$. For any given $\zeta \in S^{2 n-1}$, suppose $\max _{z \in \bar{\Omega}} \Phi(z, \zeta)$ is attained at $z_{0} \in \bar{\Omega}$.

a Case 1: $z_{0} \in \Omega$. We prove that this case will not happen with proper coefficients. Differentiating $\Phi$ at $z_{0}$, we obtain

$$
0=\Phi_{i}=h^{\prime} r_{i}\left(D_{\zeta \zeta} u-v(z, \zeta)\right)+h(r)\left(D_{\zeta \zeta} u-v(z, \zeta)\right)_{i}+u_{k} u_{\bar{k} i}+u_{\bar{k}} u_{k i},
$$

which yields that

$$
\left(D_{\zeta \zeta} u-v(z, \zeta)\right)_{i}=-\frac{h^{\prime} r_{i}\left(D_{\zeta \zeta} u-v(z, \zeta)\right)+u_{k} u_{\bar{k} i}+u_{\bar{k}} u_{k i}}{h} .
$$


Differentiating $\Phi$ a second time to get

$$
\begin{aligned}
F^{i \bar{j}} \Phi_{i \bar{j}} \geq & h^{\prime} F^{i \bar{j}} r_{i \bar{j}}\left(D_{\zeta \zeta} u-v(z, \zeta)\right) \\
& +F^{i \bar{j}} h^{\prime \prime} r_{i} r_{\bar{j}}\left(D_{\zeta \zeta} u-v(z, \zeta)\right)+h^{\prime} r_{i} F^{i \bar{j}}\left(D_{\zeta \zeta} u-v(z, \zeta)\right)_{\bar{j}} \\
& +F^{i \bar{j}} h^{\prime} r_{\bar{j}}\left(D_{\zeta \zeta} u-v(z, \zeta)\right)_{i}+h F^{i \bar{j}}\left(D_{\zeta \zeta} u-v(z, \zeta)\right)_{i \bar{j}} \\
& +F^{i \bar{j}}\left(u_{k \bar{j}} u_{\bar{k} i}+u_{\bar{k} \bar{j}} u_{k i}\right)+F^{i \bar{j}}\left(u_{k} u_{\bar{k} i \bar{j}}+u_{\bar{k}} u_{k i \bar{j}}\right),
\end{aligned}
$$

and then by (4.1), we obtain

$$
\begin{aligned}
F^{i \bar{j}} \Phi_{i \bar{j}} \geq & 2 h^{\prime} F^{i \bar{j}} r_{i}\left(-\frac{h^{\prime} r_{\bar{j}}\left(D_{\zeta \zeta} u-v(z, \zeta)\right)+u_{k} u_{\bar{k} \bar{j}}+u_{\bar{k}} u_{k \bar{j}}}{h}\right) \\
& +\left(h^{\prime} F^{i \bar{j}} r_{i \bar{j}}+F^{i \bar{j}} h^{\prime \prime} r_{i} r_{\bar{j}}\right)\left(D_{\zeta \zeta} u-v(z, \zeta)\right)+F^{i \bar{j}}\left(u_{k \bar{j}} u_{\bar{k} i}+u_{\bar{k} \bar{j}} u_{k i}\right) \\
& +F^{i \bar{j}}\left(u_{k} u_{\bar{k} i \bar{j}}+u_{\bar{k}} u_{k i \bar{j}}\right)+h F^{i \bar{j}}\left(D_{\zeta \zeta} u-v(z, \zeta)\right)_{i \bar{j}} \\
\geq & F^{i \bar{j}} r_{i} r_{\bar{j}}\left(D_{\zeta \zeta} u-v(z, \zeta)\right)\left(h^{\prime \prime}-2 \frac{\left(h^{\prime}\right)^{2}}{h}\right)-2 \frac{h^{\prime}}{h} F^{i \bar{j}} r_{i}\left(u_{k} u_{\bar{k} \bar{j}}+u_{\bar{k}} u_{k \bar{j}}\right) \\
& +h^{\prime} F^{i \bar{j}} r_{i \bar{j}}\left(D_{\zeta \zeta} u-v(z, \zeta)\right)+F^{i \bar{j}}\left(u_{k \bar{j}} u_{\bar{k} i}+u_{\bar{k} \bar{j}} u_{k i}\right)+u_{k} \tilde{\psi}_{\bar{k}}+u_{\bar{k}} \tilde{\psi}_{k} \\
& +h \tilde{\psi}_{\zeta \zeta}-h F^{i \bar{j}}\left(a_{i \bar{j}}^{l} D_{l} u+2 a_{i}^{l}\left(D_{l} u\right)_{\bar{j}}+a^{l}\left(D_{l} u\right)_{i \bar{j}}+b_{i \bar{j}}\right),
\end{aligned}
$$

where we used (2.5), $G^{i \bar{j}, k \bar{l}} \eta_{i \bar{j} \xi} \eta_{k \bar{l} \xi}+G^{i \bar{j}} \eta_{i \bar{j} \xi \xi}=\tilde{\psi}_{\xi \xi}$ and $G^{i \bar{j}, k \bar{l}} \leq 0$ in the last inequality. By Cauchy-Schwarz inequality, we see

$$
\begin{aligned}
& -2 \frac{h^{\prime}}{h} r_{i} F^{i \bar{j}}\left(u_{k} u_{\bar{k} \bar{j}}+u_{\bar{k}} u_{k \bar{j}}\right) \\
\geq & -\varepsilon F^{i \bar{j}}\left(u_{\bar{k} \bar{j}} u_{k i}+u_{k \bar{j}} u_{\bar{k} i}\right)-\frac{2}{\varepsilon}|\nabla u|^{2} \frac{\left(h^{\prime}\right)^{2}}{h^{2}} F^{i \bar{j}} r_{i} r_{\bar{j}} .
\end{aligned}
$$

Similarly, we have

$$
\begin{aligned}
& -h F^{i \bar{j}}\left(a_{i \bar{j}}^{l} D_{l} u+2 a_{i}^{l}\left(D_{l} u\right)_{\bar{j}}+a^{l}\left(D_{l} u\right)_{i \bar{j}}+b_{i \bar{j}}\right) \\
\geq & -\frac{1}{4} F^{i \bar{j}}\left(D_{l} u\right)_{\bar{j}}\left(D_{l} u\right)_{i}-C_{1}\left(h+h^{2}\right) \sum F^{i \bar{i}}-h a^{l} D_{l} \tilde{\psi} .
\end{aligned}
$$

Note that $\sum_{l=1}^{2 n} D_{l \bar{j}} u D_{l i} u=\sum_{p=1}^{n} 2 u_{p \bar{j}} u_{\bar{p} i}+2 u_{\bar{p} \bar{j}} u_{p i}$. Above all, we then arrive at

$$
\begin{aligned}
F^{i \bar{j}} \Phi_{i \bar{j}} \geq & F^{i \bar{j}} r_{i} r_{\bar{j}}\left(D_{\zeta \zeta} u-v(z, \zeta)\right)\left(h^{\prime \prime}-2 \frac{\left(h^{\prime}\right)^{2}}{h}\right)+h^{\prime} F^{i \bar{j}} r_{i \bar{j}}\left(D_{\zeta \zeta} u-v(z, \zeta)\right) \\
& +\left(\frac{1}{2}-\varepsilon\right) F^{i \bar{j}}\left(u_{k \bar{j}} u_{\bar{k} i}+u_{\bar{k} \bar{j}} u_{k i}\right)-\frac{2}{\varepsilon}|\nabla u|^{2} \frac{\left(h^{\prime}\right)^{2}}{h^{2}} F^{i \bar{j}} r_{i} r_{\bar{j}}+h \tilde{\psi}_{u} u_{\zeta \zeta} \\
& -C_{3} h-C_{2}-C_{1}\left(h+h^{2}\right) \sum F^{i \bar{i}} .
\end{aligned}
$$


By Lemma 9 and taking $\varepsilon=1 / 8$, we obtain that

$$
\begin{aligned}
F^{i \bar{j}} \Phi_{i \bar{j}} \geq & -C_{4} \times\left(D_{\zeta \zeta} u-v(z, \zeta)\right) \sum F^{i \bar{i}}-\frac{2}{\varepsilon}|\nabla u|^{2} \frac{\left(h^{\prime}\right)^{2}}{h^{2}} \sum F^{i \bar{i}} \\
& -C_{2}-C_{3} h-C_{1}\left(h+h^{2}\right) \sum F^{i \bar{i}}+\frac{c_{0}}{4} \sum_{j=1}^{n} F^{j \bar{j}} \sum_{k, i}\left(u_{k \bar{i}} u_{\bar{k} i}+u_{\bar{k} \bar{i}} u_{k i}\right) \\
\geq & -C_{4} D_{\zeta \zeta} u \sum F^{i \bar{i}}-\frac{2}{\varepsilon}|\nabla u|^{2} \frac{\left(h^{\prime}\right)^{2}}{h^{2}} \sum F^{i \bar{i}}-C_{5} \sum F^{i \bar{i}} \\
& -C_{2}-C_{3} h-C_{1}\left(h+h^{2}\right) \sum F^{i \bar{i}}+\frac{c_{0}}{4} \sum_{i=1}^{n} F^{i \bar{i}}\left(D_{\zeta \zeta} u\right)^{2}
\end{aligned}
$$

which is positive as long as $D_{\zeta \zeta} u$ is large enough. For example, it is positive when $D_{\zeta \zeta} u>D$. Here,

$$
D:=2 \sqrt{\frac{C_{4}^{2}}{c_{0}^{2}}+\frac{C_{6}}{c_{0}}}+2 \frac{C_{4}}{c_{0}},
$$

where $C_{6}=16 B^{2} A^{2}+\left(C_{1}+\frac{C_{3}}{c_{0}}\right) e^{A \max _{\Omega}|r|}+C_{1} e^{2 A \max _{\Omega}|r|}+C_{5}+\frac{C_{2}}{c_{0}} ; B=\sup _{\Omega}|\nabla u| ;$ $C_{3}$ depends on $A, \max _{\Omega}|r|$ and $\sup _{\Omega}\left(\left|\nabla^{2} r\right|+|\nabla r|\right) ; C_{4}$ depends on $\sup _{\Omega}|\nabla u|,|\nabla \phi|$ and $\partial \Omega ; C_{5}$ depends on $\sup _{\Omega}|\nabla \phi|, \beta$ and $\partial \Omega$.

Denote $\max _{(z, \zeta) \in \bar{\Omega} \times \mathbb{S}^{2 n-1}} \Phi(z, \zeta)=\Phi\left(z_{0}, \zeta_{0}\right)$. If $D_{\zeta_{0} \zeta_{0}} u\left(z_{0}\right)>D$, by the analysis in Case 1, then we know $z_{0} \in \partial \Omega$. Otherwise, we have proved this theorem. Now let us deal with Case 2.

a Case 2: $z_{0} \in \partial \Omega$. We further divide this case into two subcases according to whether the direction $\zeta_{0}$ is tangential or non-tangential to the boundary.

(a) If $\zeta_{0}$ is non-tangential at $z_{0} \in \partial \Omega$, then we can write $\zeta_{0}=\beta_{1} \tau+\beta_{2} \nu$, where $\tau \in \mathbb{S}^{2 n-1}$ is tangential at $z_{0}$, that is $\langle\tau, \nu\rangle=0, \beta_{1}=\left\langle\zeta_{0}, \tau\right\rangle, \beta_{2}=\left\langle\zeta_{0}, \nu\right\rangle \neq 0$, and $\beta_{1}^{2}+\beta_{2}^{2}=1$. Then, we have

$$
\begin{aligned}
D_{\zeta_{0} \zeta_{0}} u\left(z_{0}\right)= & \beta_{1}^{2} D_{\tau \tau} u\left(z_{0}\right)+\beta_{2}^{2} D_{\nu \nu} u\left(z_{0}\right)+2 \beta_{1} \beta_{2} D_{\tau \nu} u\left(z_{0}\right) \\
= & \beta_{1}^{2} D_{\tau \tau} u\left(z_{0}\right)+\beta_{2}^{2} D_{\nu \nu} u\left(z_{0}\right) \\
& +2\left(\xi_{0} \cdot \nu\right)\left[\xi_{0}-\left(\xi_{0} \cdot \nu\right) \nu\right] \cdot\left[D \phi-\beta D u-D_{l} u D \nu^{l}\right]
\end{aligned}
$$

from which we see

$$
\Phi\left(z_{0}, \zeta_{0}\right)=\beta_{1}^{2} \Phi\left(z_{0}, \tau\right)+\beta_{2}^{2} \Phi\left(z_{0}, \nu\right) .
$$

By the definition of $\Phi\left(z_{0}, \zeta_{0}\right)$, we know

$$
\Phi\left(z_{0}, \zeta_{0}\right) \leq \Phi\left(z_{0}, \nu\right) \leq C_{7}\left(1+\max _{\partial \Omega}\left|D_{\nu \nu} u\right|\right) .
$$

(b) If $\zeta_{0}$ is tangential at $z_{0} \in \partial \Omega$, then by (2.1) we have

$$
\begin{aligned}
0 \leq & D_{\nu} \Phi\left(z_{0}, \zeta_{0}\right) \\
= & -A\left(D_{\zeta_{0} \zeta_{0}} u-a^{l} D_{l} u-b\right)+D_{\nu} D_{\zeta_{0} \zeta_{0}} u \\
& -D_{\nu} a^{l} D_{l} u-a^{l} D_{\nu} D_{l} u-D_{\nu} b+\frac{1}{2} D_{k} u D_{\nu} D_{k} u \\
\leq & -A D_{\zeta_{0} \zeta_{0}} u+D_{\nu} D_{\zeta_{0} \zeta_{0}} u+\left[\frac{1}{2} D_{k} u-a^{k}\right] D_{\nu} D_{k} u+C_{8} .
\end{aligned}
$$


By the boundary condition, we know

$$
\begin{aligned}
D_{\nu} D_{\zeta_{0} \zeta_{0}} u & =D_{\zeta_{0} \zeta_{0}} D_{\nu} u-\left(D_{\zeta_{0} \zeta_{0}} \nu^{k}\right) D_{k} u-2\left(D_{\zeta_{0}} \nu^{k}\right) D_{\zeta_{0}} D_{k} u \\
& =D_{\zeta_{0} \zeta_{0}}(-\beta u+\phi)-\left(D_{\zeta_{0} \zeta_{0}} \nu^{k}\right) D_{k} u-2\left(D_{\zeta_{0}} \nu^{k}\right) D_{\zeta_{0}} D_{k} u \\
& \leq D_{\zeta_{0} \zeta_{0}}(-\beta u)+C_{9}-2\left(D_{\zeta_{0}} \nu^{k}\right) D_{\zeta_{0}} D_{k} u .
\end{aligned}
$$

By the same argument as Lemma 4.3 in [26], we know

$$
D_{\nu} D_{k} u \leq C_{10}\left(1+\left|D_{\nu \nu} u\right|\right)
$$

on $\partial \Omega$. Note that the proof of Lemma 4.3 is only related to the Neumann boundary condition. Also, by the similar argument in [30, we see

$$
-2\left(D_{\zeta_{0}} \nu^{k}\right) D_{\zeta_{0}} D_{k} u \leq C_{11} D_{\zeta_{0} \zeta_{0}} u,
$$

where $C_{11}=2 \max _{\partial \Omega}\left\{\left|\Pi_{i j}\right|\right\}$ and $\Pi_{i j}$ is the second fundamental form of the boundary. Therefore, we have

$$
0 \leq\left(-A+C_{11}-\beta\right) D_{\zeta_{0} \zeta_{0}} u+C_{12}\left(1+\left|D_{\nu \nu} u\right|\right)+C_{13} .
$$

Taking $A=1+2 \max _{\partial \Omega}\left\{\left|\Pi_{i j}\right|\right\}+|\beta|>C_{11}-\beta+1$, we get

$$
\Phi\left(z_{0}, \zeta_{0}\right) \leq C_{12}\left(1+\max _{\partial \Omega}\left|D_{\nu \nu} u\right|\right)+C_{13}+C_{14},
$$

where $C_{14}$ depends on $|u|_{C^{1}(\Omega)}$, $\max _{\Omega}|r|$ and $A$.

Remark 16. For general Neumann boundary data $\varphi(z, u)$, Theorem 15 still holds by replacing $v(z, \zeta)$ in the above proof with $v(z, \zeta)=2\langle\zeta, \nu\rangle\left\langle\zeta^{\prime}, D \varphi-D_{l} u D \nu^{l}\right\rangle$.

Remark 17. For $F\left(u_{i \bar{j}}\right)=\tilde{\psi}(z, u, D u)$ with $\tilde{\psi}(z, q, p) \in C^{2}\left(\Omega \times \mathbb{R} \times \mathbb{R}^{n}\right)$, Theorem 15 still holds. The main changes are to deal with $h \tilde{\psi}_{\zeta \zeta}$. By choosing $h=e^{-A_{1} r-A_{2}}$ for large $A_{2}$ such that $\left|h \tilde{\psi}_{p_{k} p_{l}}\right|<\frac{c_{0}}{8}$ and 4.1), we can also control $h \tilde{\psi}_{\zeta \zeta}$ and obtain Theorem 15 .

Now we estimate the double normal derivative on the boundary.

Theorem 18. Suppose $u \in C^{4}(\Omega) \cap C^{3}(\bar{\Omega})$ is a $k$-admissible solution to equation (1.4) or (1.6). Then, we have

$$
\max _{\partial \Omega}\left|D_{\nu \nu} u\right| \leq C
$$

where $C$ depends on $n, k, \Omega,|u|_{C^{1}},|\psi|_{C^{1}}$ and $|\varphi|_{C^{3}}$.

Proof. We adopt the idea in [37] for barrier function with minor changes to suit our needs. Denote $M=\sup _{\partial \Omega}\left|u_{\nu \nu}\right|$. As $\frac{\partial u}{\partial \nu}=\varphi(z, u)$, we construct the following auxiliary function

$$
\Phi=<D u, D r>-\varphi(z, u)+M^{-\frac{1}{2}}(<D u, D r>-\varphi(z, u))^{2}+\frac{1}{2} M r
$$

where $r$ is a defining function such that $r<0$ in $\Omega, r=0$ on $\partial \Omega$, and $\frac{\partial r}{\partial \nu}=1$ on $\partial \Omega$. Define

$$
\Omega_{\mu}:=\{z \in \Omega: d(z, \partial \Omega)<\mu\} .
$$

On $\partial \Omega$, it is obvious that $\Phi=0$. Take a small positive constant $\mu$ such that $r=-d$ on $\Omega_{\mu}$, where $d$ is the distance function to the boundary $\partial \Omega$. On $\partial \Omega_{\mu} \backslash \partial \Omega$, we see $\Phi<C_{1}-C_{2} M^{-1 / 2}-\frac{1}{2} M \mu<0$, when $M$ is large enough. Suppose

$$
\Phi\left(z_{0}\right)=\max _{\overline{\Omega_{\mu}}} \Phi .
$$


If $\Phi$ achieves its maximum in $\Omega_{\mu}$, i.e. $z_{0} \in \Omega_{\mu}$, we then have

$$
\begin{aligned}
0=\Phi_{i}\left(z_{0}\right)= & {[<D u, D r>-\varphi(z, u)]_{i}\left(1+2 M^{-\frac{1}{2}}(<D u, D r>-\varphi(z, u))\right) } \\
& +\frac{1}{2} M r_{i},
\end{aligned}
$$

and

$$
\begin{aligned}
0 \geq & \Phi_{i \bar{j}}\left(z_{0}\right) \\
= & {[<D u, D r>-\varphi(z, u)]_{i \bar{j}}\left(\left(1+2 M^{-\frac{1}{2}}(<D u, D r>-\varphi(z, u))\right)\right.} \\
& +2 M^{-\frac{1}{2}}[<D u, D r>-\varphi(x, u)]_{i}[<D u, D r>-\varphi(z, u)]_{\bar{j}} \\
& +\frac{1}{2} M r_{i \bar{j}} \\
= & {[<D u, D r>-\varphi(z, u)]_{i \bar{j}}\left(\left(1+2 M^{-\frac{1}{2}}(<D u, D r>-\varphi(z, u))\right)\right.} \\
& +\frac{2 M^{3 / 2} r_{i} r_{\bar{j}}}{4\left(1+2 M^{-\frac{1}{2}}(<D u, D r>-\varphi(z, u))\right)^{2}}+\frac{1}{2} M r_{i \bar{j}} .
\end{aligned}
$$

Note that

$$
\begin{aligned}
& F^{i \bar{j}}[<D u, D r>-\varphi(z, u)]_{i \bar{j}} \\
= & F^{i \bar{j}}\left[\left(D_{\alpha} u\right)_{i \bar{j}} D_{\alpha} r+\left(D_{\alpha} u\right)_{i}\left(D_{\alpha} r\right)_{\bar{j}}+\left(D_{\alpha} u\right)_{\bar{j}}\left(D_{\alpha} r\right)_{i}+D_{\alpha} u\left(D_{\alpha} r\right)_{i \bar{j}}\right] \\
& -F^{i \bar{j}}\left[\varphi_{z_{i} z_{\bar{j}}}+\varphi_{z_{i} u} u_{\bar{j}}+\varphi_{u z_{\bar{j}}} u_{i}+\varphi_{u u} u_{\bar{j}} u_{i}+\varphi_{u} u_{i \bar{j}}\right] \\
= & D_{\alpha} \tilde{\psi} D_{\alpha} r+2 F^{i \bar{j}}\left(D_{\alpha} u\right)_{i}\left(D_{\alpha} r\right)_{\bar{j}}+D_{\alpha} u F^{i \bar{j}}\left(D_{\alpha} r\right)_{i \bar{j}} \\
& -F^{i \bar{j}}\left[\varphi_{z_{i} z_{\bar{j}}}+\varphi_{z_{i} u} u_{\bar{j}}+\varphi_{u z_{\bar{j}}} u_{i}+\varphi_{u u} u_{\bar{j}} u_{i}+\varphi_{u} u_{i \bar{j}}\right] \\
\geq & -C_{15}(1+M) \sum_{i=1}^{n} F^{i \bar{i}}
\end{aligned}
$$

where in the last inequality we used Theorem 15 and $C_{15}$ is a constant depending on $|u|_{C^{1}(\Omega)},|\varphi|_{C^{2}}$ and $|r|_{C^{3}(\Omega)}$. Without loss of generality, we assume that

$$
M \geq 16\left(\sup _{\Omega}|D u|+\sup _{\Omega \times\left[\inf _{\Omega} u, \sup _{\Omega} u\right]} \varphi(z, u)\right)^{2} .
$$

So we have

$$
\left|M^{-\frac{1}{2}}(<D u, D r>-\varphi(z, u))\right| \leq 1 / 4 .
$$

By Theorem 15 and $|D r|^{2}=1$ on $\Omega_{\mu}$, we obtain

$$
\begin{aligned}
0 \geq & F^{i \bar{j}} \Phi_{i \bar{j}} \\
= & F^{i \bar{j}}[<D u, D r>-\varphi(z, u)]_{i \bar{j}}\left(1+2 M^{-\frac{1}{2}}(<D u, D r>-\varphi(z, u))\right) \\
& +\frac{2 M^{3 / 2} F^{i \bar{j}} r_{i} r_{\bar{j}}}{4\left(1+2 M^{-\frac{1}{2}}(<D u, D r>-\varphi(z, u))\right)^{2}}+\frac{1}{2} M F^{i \bar{j}} r_{i \bar{j}} \\
& \geq-C_{15}(1+M) \sum_{i=1}^{n} F^{i \bar{i}}+\frac{2}{9} c_{n, k} \sum_{i=1}^{n} F^{i \bar{i}} M^{3 / 2}>0,
\end{aligned}
$$


for large $M$ satisfying $\frac{2}{9} c_{n, k} M^{3 / 2}>C_{15}(1+M)$. This yields a contradiction. Hence, we can assume the maximum of $\Phi$ is achieved on $\partial \Omega_{\mu}$ and therefore on $\partial \Omega$.

By Hopf lemma, we have on $\partial \Omega$

$$
\begin{aligned}
0 & \leq \frac{\partial \Phi}{\partial \nu} \\
& =\left(r_{l} D_{\nu} u_{l}+u_{l} D_{\nu} r_{l}-D_{\nu} \varphi\right)\left(1+M^{-\frac{1}{2}}(<D u, D r>-\varphi)\right)+\frac{1}{2} M .
\end{aligned}
$$

If $\sup _{\partial \Omega}\left|u_{\nu \nu}\right|=-\inf _{\partial \Omega} u_{\nu \nu}=-u_{\nu \nu}\left(z_{1}\right)=M$, then from the above inequality we have

$$
0 \leq \frac{3}{4} u_{\nu \nu}\left(z_{1}\right)+C_{16}+\frac{1}{2} M
$$

which implies that $\sup _{\partial \Omega}\left|u_{\nu \nu}\right| \leq 4 C_{16}$.

Similarly, we can construct an auxiliary function

$$
\bar{\Phi}=<D u, D r>-\varphi(z, u)-M^{-\frac{1}{2}}(<D u, D r>-\varphi)^{2}-\frac{1}{2} M r .
$$

With similar argument, we know $\bar{\Phi}$ achieves its minimum at $\partial \Omega$. On $\partial \Omega$,

$$
\begin{aligned}
0 & \geq \frac{\partial \bar{\Phi}}{\partial \nu} \\
& =\left(r_{l} D_{\nu} u_{l}+u_{l} D_{\nu} r_{l}-D_{\nu} \varphi\right)\left(1-M^{-\frac{1}{2}}(<D u, D r>-\varphi)\right)-\frac{1}{2} M .
\end{aligned}
$$

If $\sup _{\partial \Omega}\left|u_{\nu \nu}\right|=\sup _{\partial \Omega} u_{\nu \nu}=u_{\nu \nu}\left(z_{2}\right)=M$, then from the above inequality we have

$$
0 \geq \frac{3}{4} u_{\nu \nu}\left(z_{2}\right)-C_{17}-\frac{1}{2} M
$$

which implies $\sup _{\partial \Omega}\left|u_{\nu \nu}\right| \leq 4 C_{17}$.

Remark 19. The double normal estimates on boundary hold for general $\psi(z, u, D u)$ and thus the global $C^{2}$ estimates hold.

Remark 20. The a priori estimates also hold for some general smooth symmetric functions of $n$ variables defined in a symmetric, open and convex cone $\Gamma \subset \mathbb{R}^{n}$ similar to those in [1].

\section{Proof of the Main theorem}

Proof of Theorem 1. Now we can give the proof of Theorem 11. The $C^{0}$ estimate is similar as [6] and we omit it here. Combining our $C^{1}, C^{2}$ estimates and EvansKrylov Theorem, we obtain

$$
|u|_{C^{2, \alpha}(\bar{\Omega})} \leq C
$$

for some uniform $C$ independent of the lower bound $\psi$ and $0<\alpha<1$. Applying the method of continuity (see 15], Theorem 17.28), we complete the proof of Theorem 1 .

Proof of Theorem 3. The proof of Theorem 2 is almost the same as Theorem 1.3 in Chen-Wei [6] and we sketch the proof here for the completeness. Denote $u_{\varepsilon}$ as the solution to

$$
\begin{cases}\sigma_{k}(\Delta u \mathrm{I}-\partial \bar{\partial} u)=\psi(z), & \text { in } \Omega \\ u_{\nu}=-\varepsilon u+\phi(z), & \text { on } \partial \Omega\end{cases}
$$


The existence of $u_{\varepsilon}$ holds by Theorem 1. Following the proof of Theorem 1.3 in Chen-Wei [6] closely, we can obtain $\left|\varepsilon u_{\varepsilon}\right|_{C^{0}(\Omega)} \leq C,\left|\nabla u_{\varepsilon}\right| \leq C$, and $\left|\nabla^{2} u_{\varepsilon}\right| \leq C$, where $C$ is a positive constant independent of $\varepsilon$. Here the convexity condition is to obtain the uniform $C^{1}$ estimates respect $\varepsilon$. Let

$$
v_{\varepsilon}=u_{\varepsilon}-\frac{1}{|\Omega|} \int_{\Omega} u_{\varepsilon}
$$

and satisfy

$$
\left\{\begin{array}{l}
\frac{\sigma_{n}\left(\Delta v_{\varepsilon} \mathrm{I}-\partial \bar{\partial} v_{\varepsilon}\right)}{\sigma_{l}\left(\Delta v_{\varepsilon} \mathrm{I}-\partial \bar{\partial} v_{\varepsilon}\right)}=\psi(z), \quad \text { in } \quad \Omega, \\
D_{\nu}\left(v_{\varepsilon}\right)=-\varepsilon v_{\varepsilon}-\frac{1}{|\Omega|} \int_{\Omega} \varepsilon u_{\varepsilon}+\phi(z), \quad \text { on } \quad \partial \Omega .
\end{array}\right.
$$

By the uniform global $C^{1}$ estimates, there exist a subsequence $v_{\varepsilon}$ converging to $v$ and a constant $c$ such that

$$
\left\{\begin{array}{l}
\frac{\sigma_{n}(\Delta v \mathrm{I}-\partial \bar{\partial} v)}{\sigma_{l}(\Delta v \mathrm{I}-\partial \bar{\partial} v)}=\psi(z), \quad \text { in } \quad \Omega \\
D_{\nu}(v)=c+\phi(z), \quad \text { on } \quad \partial \Omega .
\end{array}\right.
$$

The uniqueness is obtained by the Hopf lemma and same as [6].

Proof of Theorem [5. The $C^{0}$ estimate holds by the standard argument in [6]. Then Theorem 3 follows by Theorem 13, Theorem 15 and Theorem 18

Proof of Theorem Q The proof is same as Theorem 1 by deleting the bar.

\section{REFERENCES}

[1] L. A. Caffarelli, L. Nirenberg and J. Spruck, Dirichlet problem for nonlinear second order elliptic equations III, Functions of the eigenvalues of the Hessian, Acta Math. 155 (1985), 261-301.

[2] C.Q. Chen, L. Chen, X.Q. Mei and N. Xiang, Neumann problem for a class of fully nonlinear equations, arXiv:2003.05323

[3] C.Q. Chen, L. Chen, X.Q. Mei and N. Xiang, The Neumann problem for a class of mixed complex Hessian equations, arXiv:2003.06147

[4] C.Q. Chen, X.N. Ma and W. Wei, The Neumann problem of special Lagrangian equations with supercritical phase, J. Differential Equations 267 (2019), no. 9, 5388-5409.

[5] C.Q. Chen, X.N. Ma and W. Wei, The Neumann problem of complex special Lagrangian equations with supercritical phase, Anal. Theory Appl. 35 (2019), no. 2, 144-162.

[6] C.Q. Chen and W. Wei, The Neumann problem of complex Hessian quotient equations, International Mathematics Research Notices, rnaa081, 2020.

[7] C.Q. Chen and D.K. Zhang, The Neumann problem of Hessian quotient equations, Bulletin of Mathematical Sciences, accepted, 2020. DOI: 10.1142/S1664360720500186.

[8] S. Chen, Boundary value problems for some fully nonlinear elliptic equations. Cal. Var. Partial Differential Equations 30 (2007): 1-15.

[9] S. Chen, Conformal deformation on manifolds with boundary. Geom. Funct. Anal. 19 (2009), no. 4, 1029-1064.

[10] J. Chu, H. Jiao, Curvature estimates for a class of Hessian type equations, to appear in Calc. Var. Partial Differential Equations.

[11] X. J. Chen, Q. Tu, N. Xiang, A class of Hessian quotient equations in Euclidean space. J. Differential Equations 269 (2020), no. 12, 11172-11194.

[12] B. Deng, The Monge-Ampère equation for strictly $(n-1)$-convex functions with Neumann boundary. J. Math. Study 53 (2020), no. 1, 66-65.

[13] B. Deng, The Neumann problem for a class of fully nonlinear elliptic partial differential equations, arXiv:1903.04231

[14] K. Feng, H.B. Ge and T. Zheng, The Dirichlet Problem of Fully Nonlinear Equations on Hermitian Manifolds, arXiv: 1905.02412. 
[15] D. Gilbarg and N. Trudinger, Elliptic partial differential equations of second order. Second edition. Grundlehren der Mathematischen Wissenschaften [Fundamental Principles of Mathematical Sciences], 224. Springer-Verlag, Berlin, 1983. xiii+513 pp. ISBN: 3-540-13025-X.

[16] X.L. Han and X.S. Jin, limit behavior of deformed Hermitian-Yang-Mills equations with Dirichlet and Neumann boundary values conditions. preprint, 2019.

[17] Y. He, W. M. Sheng, Prescribing the symmetric function of the eigenvalues of the Schouten tensor, Proc. Amer. Math. Soc., 139(2011): 1127-1136.

[18] Y. He, W. M. Sheng, On existence of the prescribing $k$-curvature problem on manifolds with boundary, Comm. Anal. Geom. 19(2011), no. 1, 53-77.

[19] Z.L. Hou, X.N. Ma and D.M. Wu, A second order estimate for complex Hessian equations on a compact Kähler manifold, Math. Res. Lett. 17 (2010), 547-561.

[20] G. Huisken and C. Sinestrari, Convexity estimates for mean curvature flow and singularities of mean convex surfaces, Acta. math. 183 (1999), 45-70.

[21] F.D. Jiang and N.S. Trudinger, Oblique boundary value problems for augmented Hessian equations II. Nonlinear Anal. 154(2017),148-173.

[22] F.D. Jiang and N.S. Trudinger, Oblique boundary value problems for augmented Hessian equations I. Bull. Math. Sci. 8(2018), 353-411.

[23] Q. Jin, Local Hessian estimates for some conformally invariant fully nonlinear equations with boundary conditions. Differential Integral Equations 20 (2007), 121-132.

[24] Q. Jin, A.B. Li, and Y.Y. Li, Estimates and existence results for a fully nonlinear Yamabe problem on manifolds with boundary. Cal. Var. Partial Differential Equations 28 (2007): 509-543.

[25] Y. Y. Li, Some existence results of fully nonlinear elliptic equations of Monge-Ampère type, Comm. Pure Appl. Math. 43 (1990), 233-271.

[26] S.Y. Li, On the Neumann problems for complex Monge-Ampère equations, Indiana Univ. Math. J. 43 (1994), no. 4, 1099-1122.

[27] S.Y. Li, On the Dirichlet problems for symmetric function equations of the eigenvalues of the complex Hessian, Asian J. Math. 8 (2004), 87-106.

[28] P.L. Lions, N. Trudinger and J. Urbas, The Neumann problem for equations of Monge-Ampère type, Comm. Pure Appl. Math., 39(1986), 539-563.

[29] M. Lin and N.S. Trudinger, On some inequalities for elementary symmetric functions, Bull. Austral. Math. Soc., 50(1994), 317-326.

[30] X.N. Ma and G.H. Qiu, The Neumann problem for Hessian equations, Comm. Math. Phys. 366 (2019), no. 1, 1-28.

[31] G.H. Qiu and C. Xia, Classical Neumann problems for Hessian equations and AlexandrovFenchel's inequalities, Int. Math. Res. Not. IMRN 2019, no. 20, 6285-6303.

[32] W.M. Sheng, L. X. Yuan, A class of Neumann problems arising in conformal geometry, Pacific J. Math. 270 (2014), no. 1, 211-235.

[33] W.M. Sheng, L. X. Yuan, Prescribing curvature problems on the Bakry-Emery Ricci tensor of a compact manifold with boundary, Chin. Ann. Math. Ser. B 35 (2014), no. 1, 139-160.

[34] J. Spruck, Geometric aspects of the theory of fully nonlinear elliptic equations. Global theory of minimal surfaces, 283-309, Clay Math. Proc., 2, Amer. Math. Soc., Providence, RI, 2005.

[35] G. Székelyhidi, V. Tosatti and B. Weinkove, Gauduchon metrics with prescribed volume form, Acta Math. 219 (2017), 181-211.

[36] V. Tosatti and B. Weinkove, The complex Monge-Ampère equation with a gradient term, arXiv: 1906.10034 .

[37] J. Wang, The Neumann problem for complex special Lagrangian equations with critical phase, International Journal of Mathematics 30(2019), No. 9 :1950043, 28pp.

[38] J. Wang, The Neumann problem for special Lagrangian equations with critical phase, Commun. Math. Stat. 7 (2019), no. 3: 329-361.

[39] R.R. Yuan, On a class of fully nonlinear elliptic equations containing gradient terms on compact Hermitian manifolds, Canad. J. Math. 70 (2018), 943-960.

[40] R.R. Yuan, Regularity of fully nonlinear elliptic equations on Hermitian manifolds, II, arXiv:2001.09238 
School of Mathematics, Tianjin University, Tianjin, P.R.China, 300354

Email address: dr.dong@tju.edu.cn

Shanghai Center for Mathematical Sciences, Fudan University, Shanghai, P.R.China.

Email address: wei_wei@fudan.edu.cn 